\title{
Periventricular Hemorrhage of the Newborn
}

National Cancer Institute

\section{Source}

National Cancer Institute. Periventricular Hemorrhage of the Newborn. NCI Thesaurus. Code C117272.

Bleeding into the brain tissue adjacent to the lateral cerebral ventricles of newborn infant. 